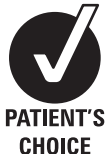

CHOICE

\title{
Smoking and the risk of amyotrophic lateral sclerosis: a systematic review and meta-analysis
}

\author{
Alvaro Alonso, ${ }^{1,2}$ Giancarlo Logroscino, ${ }^{3}$ Miguel A Hernán ${ }^{4,5}$
}

\begin{abstract}
- Additional data, tables and figures are published online only. To view these files please visit the journal online (http:// jnnp.bmj.com).

${ }^{1}$ Division of Epidemiology and Community Health, School of Public Health, University of Minnesota, Minneapolis, Minnesota, USA

${ }^{2}$ Department of Preventive Medicine and Public Health, School of Medicine, University of Navarra, Pamplona, Spain ${ }^{3}$ Department of Neurological Sciences, University of Bari, Bari, Italy

${ }^{4}$ Department of Epidemiology, Harvard School of Public Health, Boston, Massachusetts, USA

${ }^{5}$ Harvard-MIT Division of Health Sciences, Boston,

Massachusetts, USA
\end{abstract}

\section{Correspondence to}

Dr A Alonso, Division of Epidemiology and Community Health, School of Public Health, University of Minnesota, 1300 S, Suite 300, MN 55416, USA; aalogut@alumni.unav.es

Received 7 April 2009 Revised 22 June 2009 Accepted 6 July 2009 Published Online First 16 July 2010

\section{ABSTRACT}

Background Epidemiologic studies have provided inconsistent results on the association of cigarette smoking with the incidence of amyotrophic lateral sclerosis (ALS). To summarise published evidence and explore sources of heterogeneity, we conducted a systematic review and meta-analysis of studies that evaluated this association.

Methods Published studies evaluating the association of smoking with incidence of ALS were searched in bibliographic databases, with relevant information collected from each article. A random effects approach was used to pool the relative rate (RR) estimates from different studies. Between study heterogeneity was explored with a meta-regression approach.

Results 18 publications reported associations between smoking and ALS risk in 15 case control studies and five cohort studies. The pooled RR (95\% CI) of ALS was 1.28 (0.97 to 1.68$)$ for current versus never smokers and 1.12 (0.98 to 1.27 ) for ever versus never smokers. The study specifics RRs were heterogeneous $(p<0.01)$. The proportion of women in the study population explained $46 \%$ of between study variability. The estimated RR $(95 \% \mathrm{Cl})$ of ALS for ever versus never smokers was 0.86 (0.71 to 1.03 ) in men and 1.66 (1.31 to 2.10 ) in women. Interpretation This meta-analysis does not support an overall strong association of smoking with ALS risk but suggests that smoking might be associated with a higher risk of ALS in women.

\section{INTRODUCTION}

Amyotrophic lateral sclerosis (ALS) is a severe neurodegenerative disease of unknown aetiology. ${ }^{1}$ Cigarette smoking has been proposed as a potential causative factor for ALS but published epidemiologic studies have provided inconsistent results. Methodological differences and heterogeneity in studied populations could account in part for these disparities. ${ }^{2}$

We conducted a systematic review and metaanalysis of published studies to estimate more precisely the association between smoking and incidence of ALS, and to identify sources of heterogeneity across studies.

\section{METHODS}

\section{Search strategy}

We performed a systematic search of published studies in Medline (1950-April 2009), EMBASE (1980-April 2009) and ISI Web of Science (1975-April 2009) using the terms '(ALS OR amyotrophic lateral sclerosis OR motor neuron disease) AND (smok*OR tobacco OR cigar*)'. We considered studies published in any language.
Bibliographic references in the publications meeting inclusion criteria (see below) were reviewed to identify additional relevant papers.

\section{Selection criteria}

Studies meeting the following criteria were included in the review: (1) case control or cohort design; (2) information on smoking status referred to the period prior to diagnosis of ALS; (3) outcome defined as a medical diagnosis of ALS or presence of ALS in a death certificate; and (4) reporting of measures of association between smoking and ALS, or enough information to compute the association, or the corresponding author providing the necessary information on request. No specific exclusion criteria were applied.

\section{Data extraction}

From each identified eligible publication, the following information was abstracted: study design (case control, cohort), location, number of participants, period of recruitment, type of cases and controls (for case control studies), average followup (for cohort studies), method of case ascertainment, diagnostic criteria, response rate, mean age and range, proportion of men in the study sample, adjustment variables, as well as the main results (ORs or rate ratios of ALS in current smokers vs never smokers, in ever smokers vs never smokers and results by gender if available). In cases where the original publication did not provide enough information to estimate associations between smoking and ALS, the corresponding author was contacted. ${ }^{3-5}$

\section{Statistical analysis}

In case control studies with density sampling of controls, the OR estimates the rate ratio in the source population while in case control studies not using density sampling the $\mathrm{OR}$ is a good approximation to the rate ratio when incidence of disease is low, as in the case of ALS. ${ }^{6}$ Therefore, in this article we use the term rate ratio (RR) for association measures from both cohort and case control studies.

Heterogeneity of study specific RR estimates was evaluated by computing the $\mathrm{Q}$ and $\mathrm{I}^{2}$ statistics. ${ }^{7} \mathrm{I}^{2}$ is a measure of heterogeneity recommended by the Cochrane collaboration, ranging between $0 \%$ (no heterogeneity) to $100 \%$. Because of the evidence of heterogeneity, we did not adopt a fixed effects approach to pool the study specific estimates. Rather, we used the DerSimonian and Laird random effects method. ${ }^{8}$

To assess sources of heterogeneity we regressed the $\log \mathrm{RR}$ on study specific characteristics 





(percentage of men in the study population, average age of participants, study design and type of case-prevalent, incident or ALS death). Each study was weighted by the inverse of the study specific variance. This meta-regression model was fit separately by study design (case control vs cohort) and also in the entire sample of studies. Publication bias was assessed visually with a funnel plot.

\section{RESULTS}

The search identified 96 publications in Medline, 49 in EMBASE and 153 in ISI Web of Science. Sixteen publications met the inclusion criteria. ${ }^{3} 4$ 9-22 Additionally, we included two conference abstracts not yet published as original articles. ${ }^{523}$ Fourteen references corresponded to case control studies (one of them included results from two different studies) ${ }^{10}$ and four to prospective cohorts. One of the cohorts provided separate results for men and women. ${ }^{18}$ We considered them as separate studies since they found different results by gender. Table 1 reports the main characteristics for studies included in the meta-analysis.

Seven case control studies and five cohorts provided associations comparing the risk of ALS in current smokers versus never smokers (figure 1). There was evidence of between study heterogeneity (case control studies: $\mathrm{Q}=14.54, \mathrm{p}=0.02, \mathrm{I}^{2}=59 \%$; cohort studies: $\mathrm{Q}=25.62, \mathrm{p}<0.001, \mathrm{I}^{2}=84 \%$; pooled results: $\left.\mathrm{Q}=41.76, \mathrm{p}<0.001, \mathrm{I}^{2}=74 \%\right)$. The pooled $\mathrm{RR}$ and $95 \% \mathrm{CI}$ of ALS in current smokers versus never smokers was 1.42 (0.96 to 2.11) in case control studies and 1.16 (0.78 to 1.73$)$ in cohort studies. The pooled RR $(95 \% \mathrm{CI})$ including case control and cohort studies was 1.28 (0.97 to 1.68 ).

Fifteen case control studies and five cohorts compared the risk of ALS in ever smokers versus never smokers (figure 1). No association was evident in cohort (pooled RR 1.13, 95\% CI 0.86

Current smoker vs. never smoker

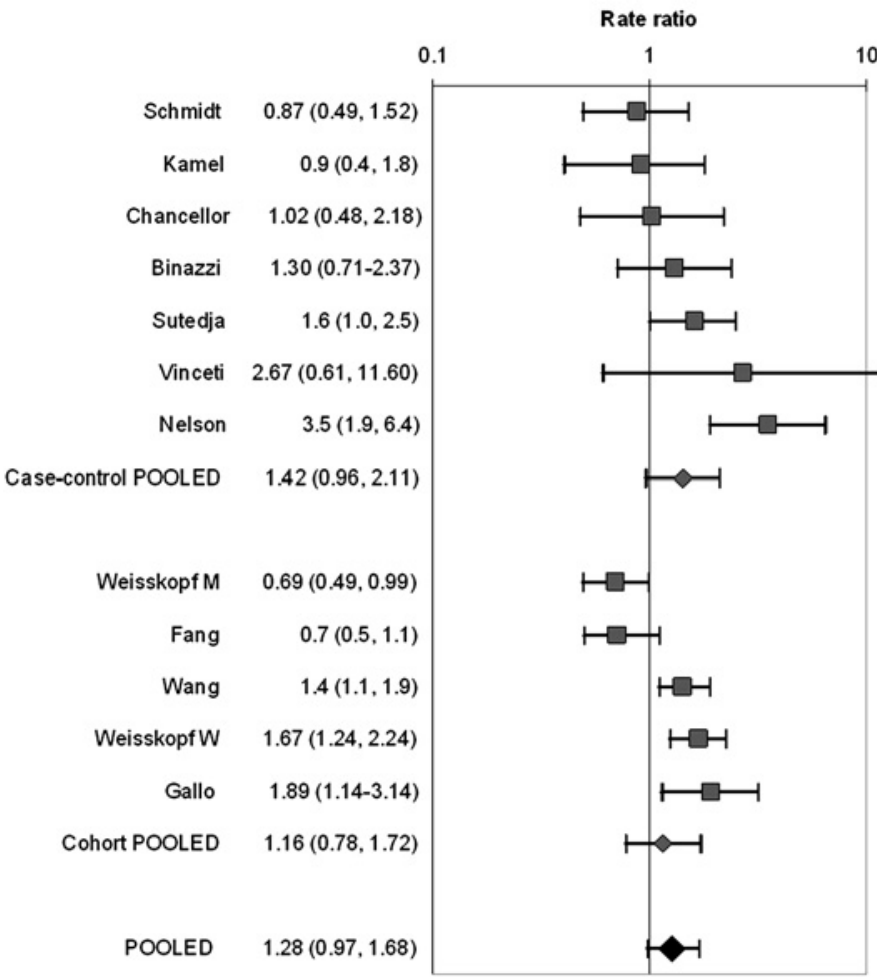

to 1.49) or in case control studies (pooled RR 1.09, 95\% CI 0.95 to 1.26$)$. The pooled RR ( $95 \% \mathrm{CI}$ ) for cohort and case control studies was $1.12(0.98,1.27)$. There was substantial evidence of between study heterogeneity $\left(\mathrm{Q}=44.74, \mathrm{p}<0.001, \mathrm{I}^{2}=58 \%\right.$ for pooled case control and cohort studies).

A few studies evaluated the existence of a dose-response trend in the association between smoking and risk of ALS. Four of them reported evidence of a trend in the association between smoking amount and risk of ALS. ${ }^{14} 152123$ Other studies, though, did not find any clear dose-response associations. ${ }^{5} 17-19$ The amount of information was insufficient to conduct a metaregression analysis of dose-response.

We tested whether study specific characteristics contributed to explain the heterogeneity across studies. In a meta-regression model, the proportion of women was the only major predictor of the association between smoking and ALS incidence: a 10\% increase in the proportion of women was significantly associated with an increase of $7 \%$ (95\% CI 3\% to 11\%) in the RR between ever smoking and ALS. The proportion of women explained $46 \%$ of the total variability in the log RR. The meta-regression predicted an RR of ALS for ever smokers versus never smokers of 0.86 (95\% CI 0.71, 1.03) in men and 1.66 (95\% CI 1.31, 2.10) in women (see web figure 1, available online). Mean age of the study participants was weakly associated with the study specific risk ratios. The study specific $\mathrm{RR}$ of the association between ever smoking and ALS increased 5\% (95\% CI -12\% to $27 \%$ ) per each 5 year increase in the average age of study participants. Other variables, such as study design or type of case (prevalent, incident, mortality) did not explain substantial between study heterogeneity (see web table 1, available online). A funnel plot of studies that estimated the association for ever versus never smokers did not indicate publication bias (web figure 2, available online).

\section{Ever smoker vs. never smoker}

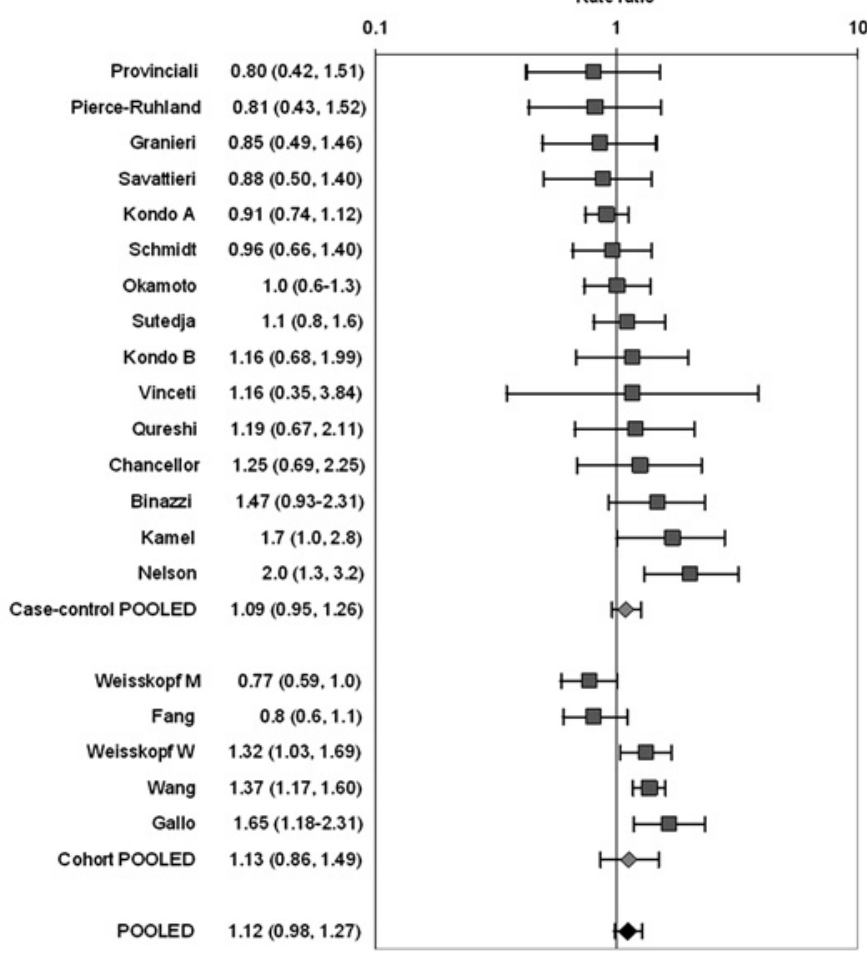

Figure 1 Study specific and pooled rate ratios of smoking (current smokers vs never smokers; ever smokers vs never smokers) and amyotrophic lateral sclerosis in published case control and cohort studies. 


\section{DISCUSSION}

This meta-analysis does not support an overall strong association of smoking with ALS risk but suggests that smoking might be associated with a higher risk of ALS in women.

Smoking could increase the risk of ALS through several mechanisms. Cigarette smoke contains a large amount of oxidant compounds which target certain molecules such as fatty acids in cell membranes and reduces the antioxidant capacity of the organism. ${ }^{24}$ For example, 8-hydroxy-2'-deoxyguanosine, a well established marker of oxidative damage to DNA, is increased in smokers compared with non-smokers, ${ }^{25}$ and its levels were higher in patients with sporadic ALS than in controls. $^{26} 27$ Also, numerous chemicals, some of them with potential neurotoxic effects, abound in cigarette smoke. Lead and formaldehyde, both present in cigarette smoke, have been associated with the risk of ALS in some studies. ${ }^{28} 29$

The stronger association between smoking and ALS in women could be explained by differences in the metabolism of chemicals present in cigarette smoke. Many smoke components are metabolised by oxidation followed by conjugation. Some studies have shown that oxidation, but not conjugation, is upregulated in women, which leads to the accumulation of intermediate metabolites and increased oxidative stress. ${ }^{30}$ The association between smoking and other health outcomes, including thyroid disease, lung function and multiple sclerosis, is modified by sex. $^{31-33}$

The present meta-analysis has some limitations. The methodological quality of the included studies was not uniform. Some studies had an unclear definition of the outcome, potentially biased selection of controls, greater opportunities for measurement error in smoking assessment and insufficient control for confounding. However, methodological quality is unlikely to explain our findings as the results did not differ between cohort and case control studies even though cohort studies were of better methodological quality overall. As in any meta-analysis, publication bias could be present but there is no clear evidence of it.

We recommend that future studies on smoking and ALS provide sex specific estimates of the association while recognising that stratified analysis of any individual study will have limited statistical power (see supplemental data, available online).

Acknowledgements The authors thank Andrew Chancellor, Enrico Granieri and Silke Schmidt who kindly provided additional unpublished information from some of the studies included in the meta-analysis.

Competing interests None.

Provenance and peer review Not commissioned; externally peer reviewed.

\section{REFERENCES}

1. Armon C. Amyotrophic lateral sclerosis. In: Nelson LM, Tanner CM, Van Den Eeden SK, et al. eds. Neuroepidemiology from principles to practice. New York: Oxford University Press, 2004:162-87.

2. Armon C. An evidence-based medicine approach to the evaluation of the role of exogenous risk factors in sporadic amyotrophic lateral sclerosis. Neuroepidemiology 2003;22:217-28.

3. Granieri $\mathbf{E}$, Carreras $\mathrm{M}$, Tola $\mathrm{R}$, et al. Motor neuron disease in the province of Ferrara, Italy, in 1964-1982. Neurology 1988;38:1604-8.

4. Chancellor AM, Slattery JM, Fraser $\mathrm{H}$, et al. Risk factors for motor neuron disease: a case-control study based on patients from the Scottish Motor Neuron Disease Register. J Neurol Neurosurg Psychiatry 1993;56:1200-6.
5. Schmidt S, Allen K, Rimmler J, et al. Do head injury or cigarette smoking contribute to the increased risk of amyotrophic lateral sclerosis in US veterans? Neuroepidemiology 2008;30:134.

6. Greenland S, Rothman KJ, Lash TL. Measures of effect and measures of association. In: Rothman KJ, Greenland S, Lash TL, eds. Modern epidemiology, 3rd edn. Philadelphia: Lippincott Williams \& Wilkins, 2008:51-70.

7. Higgins JP, Thompson SG, Deeks JJ, et al. Measuring inconsistency in metaanalyses. BMJ 2003;327:557-60.

8. DerSimonian R, Laird N. Meta-analysis in clinical trials. Control Clin Trials 1986; 7:177-88.

9. Pierce-Ruhland R, Pattern BM. Repeat study of antecedent events in motor neuron disease. Ann Clin Res 1981;13:102-7.

10. Kondo K, Tsubaki T. Case-control studies of motor neuron disease: associations with mechanical injuries. Arch Neurol 1981;38:220-6.

11. Provinciali L, Giovagnoli AR. Antecedent events in amyotrophic lateral sclerosis: do they influence clinical onset and progression? Neuroepidemiology 1990;9:255-62.

12. Savettieri G, Salemi G, Arcara A, et al. A case-control study of amyotrophic lateral sclerosis. Neuroepidemiology 1991;10:242-5.

13. Vinceti M, Guidetti D, Bergomi M, et al. Lead, cadmium, and selenium in the blood of patients with sporadic amyotrophic lateral sclerosis. Ital J Neurol Sci 1997; 18:87-92.

14. Kamel F, Umbach DM, Munsat TL, et al. Association of cigarette smoking with amyotrophic lateral sclerosis. Neuroepidemiology 1999;18:194-202.

15. Nelson LM, McGuire V, Longstreth WT Jr, et al. Population-based case-control study of amyotrophic lateral sclerosis in western Washington State. I. Cigarette smoking and alcohol consumption. Am J Epidemiol 2000;151:156-63.

16. Qureshi MM, Hayden D, Urbinelli L, et al. Analysis of factors that modify susceptibility and rate of progression in amyotrophic lateral sclerosis (ALS). Amyotroph Lateral Scler 2006; 7:173-82.

17. Sutedja NA, Veldink JH, Fischer K, et al. Lifetime occupation, education, smoking and risk of ALS. Neurology 2007;69:1508-14.

18. Weisskopf MG, McCullough ML, Calle EE, et al. Prospective study of cigarette smoking and amyotrophic lateral sclerosis. Am J Epidemiol 2004;160:26-33.

19. Fang $\mathbf{F}$, Bellocco R, Hernán MA, et al. Smoking, snuff dipping and the risk of amyotrophic lateral sclerosis-a prospective cohort study. Neuroepidemiology 2006;27:217-21.

20. Okamoto K, Kihira T, Kondo T, et al. Lifestyle factors and risk of amyotrophic latera sclerosis: a case-control study in Japan. Ann Epidemiol 2009;19:359-64.

21. Gallo V, Bueno-De-Mesquita HB, Vermeulen R, et al. Smoking and risk for amyotrophic lateral sclerosis: analysis of the EPIC cohort. Ann Neurol 2009:65:378-85.

22. Binazzi A, Belli S, Uccelli $R$, et al. An exploratory case-control study on spinal and bulbar forms of amyotrophic lateral sclerosis in the province of Rome. Amyotroph Lateral Scler 2009;10:361-9.

23. Wang H, Weisskopf MG, O'Reilly E, et al. Prospective studies on smoking and risk of amyotrophic lateral sclerosis. Neurology 2008;70(Suppl 1):A190.

24. Yanbaeva DG, Dentener MA, Creutzberg EC, et al. Systemic effects of smoking. Chest 2007;131:1557-66.

25. Loft S, Vistisen $\mathrm{K}$, Ewertz $\mathrm{M}$, et al. Oxidative DNA damage estimated by 8hydroxydeoxyguanosine excretion in humans: influence of smoking, gender and body mass index. Carcinogenesis 1992;13:2241-7.

26. Ferrante RJ, Browne SE, Shinobu LA, et al. Evidence of increased oxidative damage in both sporadic and familial amyotrophic lateral sclerosis. J Neurochem 1997:69:2064-74.

27. Bogdanov M, Brown RH Jr, Matson W, et al. Increased oxidative damage to DNA in ALS patients. Free Radic Biol Med 2000;29:652-8.

28. Kamel F, Umbach DM, Hu H, et al. Lead exposure as a risk factor for amyotrophic lateral sclerosis. Neurodegener Dis 2005;2:195-201.

29. Weisskopf MG, Morozova N, O'Reilly EJ, et al. Prospective study of chemical exposures and amyotrophic lateral sclerosis. J Neurol Neurosurg Psychiatry 2009;80:558-61

30. Sin DD, Cohen SB, Day A, et al. Understanding the biological differences in susceptibility to chronic obstructive pulmonary disease between men and women. Proc Am Thorac Soc 2007;4:671-4.

31. Vestergaard P. Smoking and thyroid disorders - a meta-analysis. Eur J Endocrino 2002:146:153-61.

32. Gan W, Man SF, Postma D, et al. Female smokers beyond the perimenopausal period are at increased risk of chronic obstructive pulmonary disease: a systematic review and meta-analysis. Respir Res 2006;7:52.

33. Sundstrom $\mathbf{P}$, Nystrom L, Hallmans G. Smoke exposure increases the risk for multiple sclerosis. Eur J Neurol 2008:15:579-83.

34. Schmidt S, Allen KD, Loiacono VT, et al. Genes and environmental exposures in veterans with amyotrophic lateral sclerosis: the GENEVA Study. Rationale, study design and demographic characteristics. Neuroepidemiology 2008;30:191-204. 